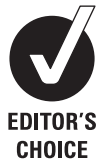

- Additional data are published online only. To view these files please visit the journal online (http://bjsm.bmj.com/ content/46/5.toc)

1 Department of Family Medicine, University of Washington, Seattle, Washington, USA 2Division of Cardiology, University of Washington, Seattle, Washington, USA ${ }^{3}$ Division of Cardiology, Seattle Children's Hospital, Seattle, Washington, USA

\section{Correspondence to} Jonathan A Drezner, MD, Department of Family Medicine, 4060 East Stevens Way, University of Washington, Box 354410, Seattle, WA 98195, USA; jdrezner@fammed. washington.edu

Received 21 September 2011 Accepted 6 January 2012

Published Online First

5 February 2012

\title{
Accuracy of ECG interpretation in competitive athletes: the impact of using standardised ECG criteria
}

\author{
Jonathan A Drezner, ${ }^{1}$ Irfan M Asif,, David S Owens, ${ }^{2}$ Jordan M Prutkin, ${ }^{2}$ \\ Jack C Salerno, ${ }^{3}$ Robyn Fean, ${ }^{1}$ Ashwin L Rao, ${ }^{1}$ Karen Stout, ${ }^{2}$ Kimberly G Harmon ${ }^{1}$
}

\begin{abstract}
Background Interpretation of ECGs in athletes is complicated by physiological changes related to training. The purpose of this study was to determine the accuracy of ECG interpretation in athletes among different physician specialties, with and without use of a standardised ECG criteria tool.
\end{abstract}

Methods Physicians were asked to interpret 40 ECGs (28 normal ECGs from college athletes randomised with 12 abnormal ECGs from individuals with known cardiovascular pathology) and classify each ECG as (1) 'normal or variant - no further evaluation and testing needed' or (2) 'abnormal - further evaluation and testing needed.' After reading the ECGs, participants received a two-page ECG criteria tool to guide interpretation of the ECGs again.

Results A total of 60 physicians participated: 22 primary care (PC) residents, $16 \mathrm{PC}$ attending physicians, 12 sports medicine (SM) physicians and 10 cardiologists. At baseline, the total number of ECGs correctly interpreted was $\mathrm{PC}$ residents $73 \%$, PC attendings $73 \%$, SM physicians $78 \%$ and cardiologists $85 \%$. With use of the ECG criteria tool, all physician groups significantly improved their accuracy ( $<<0.0001)$ : PC residents 92\%, PC attendings 90\%, SM physicians $91 \%$ and cardiologists $96 \%$. With use of the ECG criteria tool, specificity improved from $70 \%$ to $91 \%$, sensitivity improved from $89 \%$ to $94 \%$ and there was no difference comparing cardiologists versus all other physicians $(p=0.053)$.

Conclusions Providing standardised criteria to assist ECG interpretation in athletes significantly improves the ability to accurately distinguish normal from abnormal findings across physician specialties, even in physicians with little or no experience.

\section{INTRODUCTION}

The interpretation of a resting 12-lead ECG in athletes is challenging whether performed during preparticipation cardiovascular screening or for diagnostic purposes in the setting of concerning symptoms or family history. Cardiac adaptation and remodelling from regular athletic training produces common ECG alterations that may be considered abnormal in other settings. Over the past decade, criteria to differentiate normal, physiological ECG changes in athletes from ECG findings suggestive of underlying cardiovascular pathology have been refined. ${ }^{1-8}$ The use of modern, standardised ECG criteria has decreased the false-positive rate during preparticipation screening from $7-15 \%{ }^{19}$ to $2-5 \% 1011$ when performed by physicians experienced in ECG interpretation.
The European Society of Cardiology (ESC) and the International Olympic Committee (IOC) recommend a resting ECG in addition to a standard history and physical evaluation during preparticipation screening, while the American Heart Association (AHA) supports performing an ECG in the setting of concerning symptoms, family history or physical examination findings. ${ }^{3} 1213$ Whether obtained for screening or diagnostic purposes, it is critical that ECGs in athletes are correctly interpreted to avoid missing potentially dangerous cardiovascular conditions or ordering unnecessary follow-up testing. The purpose of this study was to assess the accuracy of ECG interpretation in athletes among different physician specialties, with and without use of a standardised ECG criteria tool.

\section{METHODS}

An online ECG interpretation exercise was designed consisting of 40 ECGs: 28 normal ECGs acquired from Division I college athletes randomised together with 12 abnormal ECGs from individuals with known cardiovascular pathology. The abnormal ECGs represented the most common causes of sudden cardiac death (SCD) in young athletes including five ECGs with changes consistent with hypertrophic cardiomyopathy (HCM), two demonstrating long OT syndrome, two with WolffParkinson-White syndrome, and one each with arrhythmogenic right ventricular cardiomyopathy, left ventricular non-compaction and Brugada syndrome. The normal ECGs came from National Collegiate Athletic Association (NCAA) Division I collegiate football and basketball athletes and demonstrated common ECG changes consistent with physiological adaptations of training such as sinus bradycardia, sinus arrhythmia, early repolarisation and isolated increases in ORS voltage which are considered normal based on modern criteria. The ECGs selected for inclusion were agreed upon by a panel of physicians composed of four cardiologist including a paediatric and adult electrophysiologist and two cardiomyopathy experts and three sports medicine physicians, all with experience in interpreting ECGs in athletes.

All identifying patient information and computer-generated interpretations were removed from the ECGs; however, interval values and axis measurements were left in place. The ECG interpretations provided by commercially available ECG systems are often inaccurate when applied 
Table 1 Abnormal ECG criteria in athletes. Any abnormal finding is considered training-unrelated and suggests the possibility of underlying pathological cardiac disease, requiring further diagnostic investigation.

\begin{tabular}{|c|c|}
\hline Abnormal ECG finding & Definition \\
\hline T-wave inversion & $>1 \mathrm{~mm}$ in depth from baseline in two or more adjacent leads not including aVR or V1 \\
\hline ST-segment depression & $\geq 1 \mathrm{~mm}$ in depth in two or more adjacent leads \\
\hline Pathological 0 waves & $>3 \mathrm{~mm}$ in depth or $>0.04 \mathrm{~s}$ in duration in two or more leads \\
\hline Complete left bundle branch block & QRS $>0.12 \mathrm{~s}$, predominantly negative QRS complex in lead $V_{1}\left(\mathrm{QS}\right.$ or $\mathrm{rS}$ ), and upright monophasic $\mathrm{R}$ wave in leads I and $\mathrm{V}_{6}$ \\
\hline Complete right bundle branch block & QRS $>0.12 \mathrm{~s}$, terminal $\mathrm{R}$ wave in lead $\mathrm{V}_{1}\left(\mathrm{rsR}^{\prime}\right)$ and wide terminal $\mathrm{S}$ wave in leads I and $\mathrm{V}_{6}$ \\
\hline Intraventricular conduction delay & Non-specific, QRS $>0.12 \mathrm{~s}$ \\
\hline Left atrial enlargement & $\begin{array}{l}\text { Prolonged } \mathrm{P} \text { wave duration of }>0.12 \mathrm{~s} \text { in leads I or II with negative portion of the } \mathrm{P} \text { wave } \geq 1 \mathrm{~mm} \text { in depth and } \geq 0.04 \mathrm{~s} \text { in duration in } \\
\text { lead } \mathrm{V}_{1}\end{array}$ \\
\hline Left axis deviation & $-30^{\circ}$ to $90^{\circ}$ \\
\hline Right atrial enlargement & High/pointed $\mathrm{P}$ wave $\geq 2.5 \mathrm{~mm}$ in leads II and III or $\mathrm{V}_{1}$ \\
\hline Right ventricular hypertrophy & Right axis deviation $\geq 120^{\circ}$, tall $R$ wave in $V 1+$ persistent precordial $S$ waves $\left(R-V_{1}+S-V_{5}>10.5 \mathrm{~mm}\right)$ \\
\hline Mobitz type II $2^{\circ}$ AV block & Intermittently non-conducted P waves not preceded by PR prolongation and not followed by PR shortening \\
\hline $3^{\circ} \mathrm{AV}$ block & Complete heart block \\
\hline Ventricular pre-excitation & PR interval $<0.12 \mathrm{~s}$ with a $\delta$ wave (slurred upstroke in the QRS complex) \\
\hline Long QT interval & $\begin{array}{l}\mathrm{OTc} \geq 0.47 \text { s ( } 99 \% \text { men) } \\
\mathrm{OTc} \geq 0.48 \mathrm{~s} \text { (99\% women) } \\
\text { (OTc } \geq 0.50 \text { s (unequivocal LOTS) }\end{array}$ \\
\hline Short QT interval & $0 \mathrm{Tc} \leq 0.34 \mathrm{~s}$ \\
\hline Brugada-like ECG pattern & High take-off and downsloping ST-segment elevation in $\mathrm{V}_{1}-\mathrm{V}_{3}$ \\
\hline Epsilon wave & Small negative deflection just beyond the $\mathrm{QRS}$ in $\mathrm{V}_{1}$ or $\mathrm{V}_{2}$ \\
\hline Profound sinus bradycardia & $<30 \mathrm{BPM}$ or sinus pauses $\geq 3 \mathrm{~s}$ \\
\hline Atrial tachyarrhythmias & Supraventricular tachycardia, atrioventricular nodal re-entrant tachycardia, atrial fibrillation, atrial flutter \\
\hline Premature ventricular contractions & $\geq 2$ per tracing \\
\hline Ventricular arrhythmias & Couplets, triplets, non-sustained ventricular tachycardia \\
\hline
\end{tabular}

$\mathrm{AV}$, atrioventricular; BPM, beats per minute; LQTS, long QT syndrome.

in young athletes. These ECG systems are programmed with adult and paediatric norms that do not account for the ECG changes that correspond with normal, physiological cardiac remodelling in trained athletes. Thus, computer-generated ECG interpretations will frequently list ECG findings as 'abnormal' when they are actually normal variants in athletes. Therefore, the device-generated ECG interpretations were removed to avoid biasing study participants.

Physicians from academic and community practice settings were recruited to participate from different specialties including primary-care residents, primary-care attending physicians, primary-care sports medicine attending physicians and cardiologists, none of which had any specialty training or experience in ECG interpretation in athletes. Participants were asked to assume that the ECGs were from asymptomatic athletes between the ages of 14 and 35 and to classify each ECG as either "normal or variant - no further evaluation or testing needed' or 'abnormal - further evaluation and testing needed'. After reviewing and classifying the 40 ECGs, physicians received a two-page ECG criteria tool to guide their interpretations while reviewing the 40 ECGs a second time (see Web only data supplement file). The ECG criteria tool was based on the 2010 ESC consensus statement for interpretation of the 12-lead ECG in athletes, as well as other research and input from experts familiar with cardiac screening in athletes. ${ }^{248}$ The first page of the criteria tool consisted of two tables. The first table listed each individual ECG criteria that should be considered abnormal, unrelated to athletic training, and warrant additional investigation (table 1). A second table listed the normal, physiological ECG changes commonly found in trained athletes that should not trigger additional testing (table 2). The second page of the criteria tool consisted of several figures serving as examples of uncommon ECG patterns such as a variant pattern of repolarisation in African-American athletes, complete bundle branch blocks, ventricular pre-
Table 2 Common ECG findings in athletes. Training-related ECG alterations are common, physiological adaptations to regular exercise and are considered normal variants in athletes

\begin{tabular}{l} 
Normal ECG finding \\
Sinus bradycardia \\
Sinus arrhythmia \\
First-degree AV block \\
Incomplete RBBB \\
Early repolarisation \\
Isolated QRS voltage criteria for LVH* \\
\hline *Isolated increases in QRS amplitude are common in trained athletes. However, QRS \\
voltage criteria for LVH+any non-voltage criteria for LVH (such as atrial enlargement, \\
left axis deviation, a'strain' pattern of repolarisation, ST-segment depression, T-wave \\
inversion or pathological 0 waves) are abnormal and require further evaluation. \\
AV, atrioventricular; LVH, left ventricular hypertrophy; RBBB, right bundle branch \\
block. \\
excitation, calculation of the QT interval corrected for heart rate, \\
Brugada pattern ECG and Epsilon waves. The criteria tool could \\
not be downloaded until the first round reviewing ECGs was \\
completed. During the second round of ECG review with access \\
to the criteria tool, if the ECG was classified as abnormal, the \\
participants were additionally asked to check which individual \\
criterion was present that categorised the ECG as abnormal. \\
Paired t tests were used to compare the proportion of ECGs \\
correctly categorised (normal vs abnormal) before and after use \\
of the ECG criteria guideline. Student t test was used to com- \\
pare the proportion of ECGs correctly categorised between \\
different physician specialty groups; $\alpha=0.05$ and B=0.20 \\
(power=0.80) were assumed with at least 10 physicians in each \\
specialty group needed to achieve adequate power (80\%) to \\
detect a statistically significant difference (p<0.05) in correctly \\
classifying an ECG between physician specialty groups. The \\
study was not designed to compare the correct classification
\end{tabular}
study was not designed to compare the correct classification 
of ECGs for a specific diagnosis among different physician specialties or to compare the correct classification of normal ECGs based on different findings considered normal variants. Descriptive statistics were used to analyse the classification of normal and abnormal ECGs representing a specific diagnosis by the group as a whole. The study was approved by the Human Subjects Division at the University of Washington.

\section{RESULTS}

Sixty physicians completed the exercise: 22 primary-care residents, 16 primary-care attending physicians, 12 sports medicine attending physicians and 10 cardiologists. None of the physicians had any specialty training in ECG interpretation in athletes. At baseline, primary-care residents accurately categorised $73 \%$ of ECGs as normal (no further testing needed) or abnormal (further testing needed). Primary-care attending physicians correctly classified $73 \%$, sports medicine attending physicians $78 \%$ and cardiologists $85 \%$. Cardiologists were significantly better than all primary-care physicians at interpreting the ECGs before the use of the ECG criteria tool $(p<0.001)$.

With use of the ECG criteria tool, all physician groups significantly improved their ability to correctly classify ECGs $(\mathrm{p}<0.0001)$ (figure 1). Primary-care residents increased their accuracy to $92 \%$, primary-care attending physicians to $90 \%$, sports medicine attending physicians to $91 \%$ and cardiologists to $96 \%$. With use of the ECG criteria tool, there was no statistical difference in the accuracy of ECG interpretation between cardiologists $(96 \%)$ and all other physicians (91\%) $(p=0.053)$.

The specificity of the whole group (normal ECGs correctly classified as normal) improved from $70 \%$ to $91 \%$, and the sensitivity (abnormal ECGs correctly classified as abnormal) improved from $89 \%$ to $94 \%$ with use of the ECG criteria tool. Similarly, the positive-predictive value improved from $69 \%$ to $91 \%$, and the negative-predictive value improved from $86 \%$ to $94 \%$ with use of a standardised criteria tool (table 3 ).

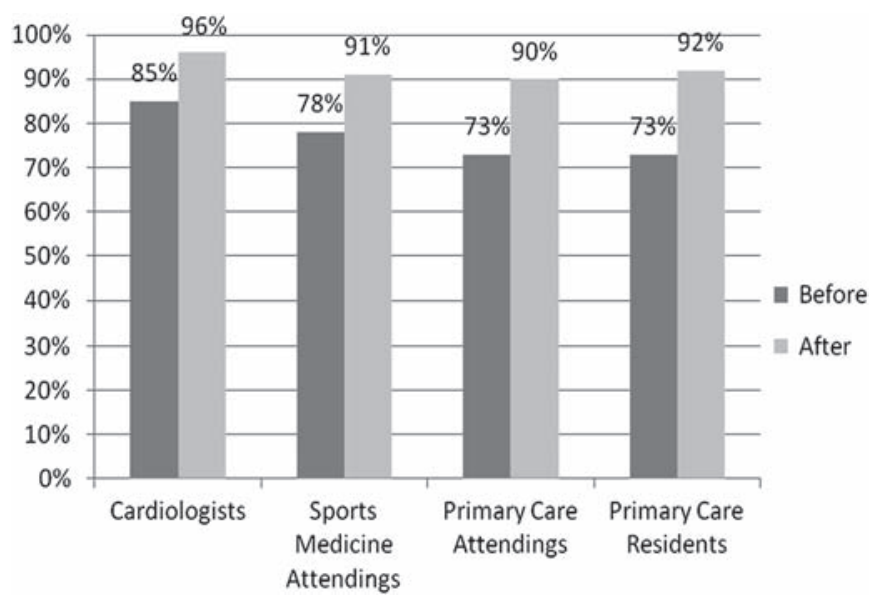

Figure 1 Accuracy of ECG interpretation by specialty before and after criteria tool.
The normal ECGs were most often incorrectly categorised. Without use of the criteria tool, a false-positive interpretation was provided in $30 \%$ of the normal ECGs. After use of the criteria tool, the false-positive rate decreased to $9 \%$. The specific criteria marked as abnormal when the ECG was actually normal varied widely. The proportion of ECGs representing a specific diagnosis that was correctly classified before and after use of the criteria tool is shown in figure 2.

\section{DISCUSSION}

SCD is the leading cause of death in young athletes on the playing field and typically the result of undiagnosed structural or electrical cardiovascular disease. ${ }^{14-16}$ The prevalence of cardiovascular disorders known to cause SCD in young athletes is approximately $0.3 \%$ or 3 athletes in $1000 .{ }^{1101317-20} \mathrm{~A}$ recent study found the incidence of SCD in college athletes to be four to five times higher than prior estimates, affecting 1 in 43000 athletes per year. ${ }^{14}$ With each tragic death of a young athlete during sports, intense public and medical scrutiny is raised regarding the adequacy of preparticipation screening programmes and current strategies for prevention.

Cardiovascular screening in athletes is routinely practiced and endorsed by most major sporting and medical associations including the AHA, ESC and IOC. ${ }^{3} 1213$ However, universal agreement on a single screening protocol to identify athletes at risk for SCD remains elusive and a topic of considerable debate. The screening controversy is centred upon the inclusion (or not) of a resting 12-lead ECG in addition to a history and physical examination during the preparticipation evaluation. While ECG increases the sensitivity to detect silent cardiac conditions in athletes, widespread use of ECG has raised concerns regarding false-positive results, cost-effectiveness, physician infrastructure and healthcare resources. ${ }^{21}$

ECG is also commonly obtained in athletes with cardiovascular-related symptoms, concerning medical history or physical examination findings. Whether performed for diagnostic or screening purposes as part of the cardiac evaluation in athletes, quality ECG interpretations are crucial to identify athletes that require additional evaluation and to limit the number of false-positive analyses that lead to expensive secondary investigations.

It is imperative to recognise that the total-positive and falsepositive rate for ECG interpretation are significantly affected by the criteria chosen to define 'abnormal.' Many ECG changes once referred to as 'abnormal' are now recognised as physiological and part of benign cardiac adaptations in athletes (socalled athlete's heart). Physicians interpreting ECGs in athletes should be familiar with common training-related ECG alterations that are normal variants. In contrast, training-unrelated ECG changes suggest the possibility of underlying pathology, require further diagnostic investigation and should be considered abnormal. In 2010, the ESC Section on Sports Cardiology published an international position statement summarising modern recommendations to distinguish pathological ECG

Table 3 Statistical measures of performance before and after criteria tool

\begin{tabular}{lllll}
\hline Physician groups & Sensitivity (before) & Sensitivity (after) & Specificity (before) & Specificity (after) \\
\hline Cardiologists & $94.2 \%$ & $98.3 \%$ & $81.1 \%$ & $94.6 \%$ \\
Sports medicine & $91.0 \%$ & $94.4 \%$ & $72.3 \%$ & $89.6 \%$ \\
Primary-care attendings & $87.0 \%$ & $95.3 \%$ & $66.7 \%$ & $87.7 \%$ \\
Primary-care residents & $87.9 \%$ & $91.3 \%$ & $66.1 \%$ & $91.6 \%$ \\
All physicians & $89.3 \%$ & $94.2 \%$ & $70.0 \%$ & $90.7 \%$ \\
\hline
\end{tabular}




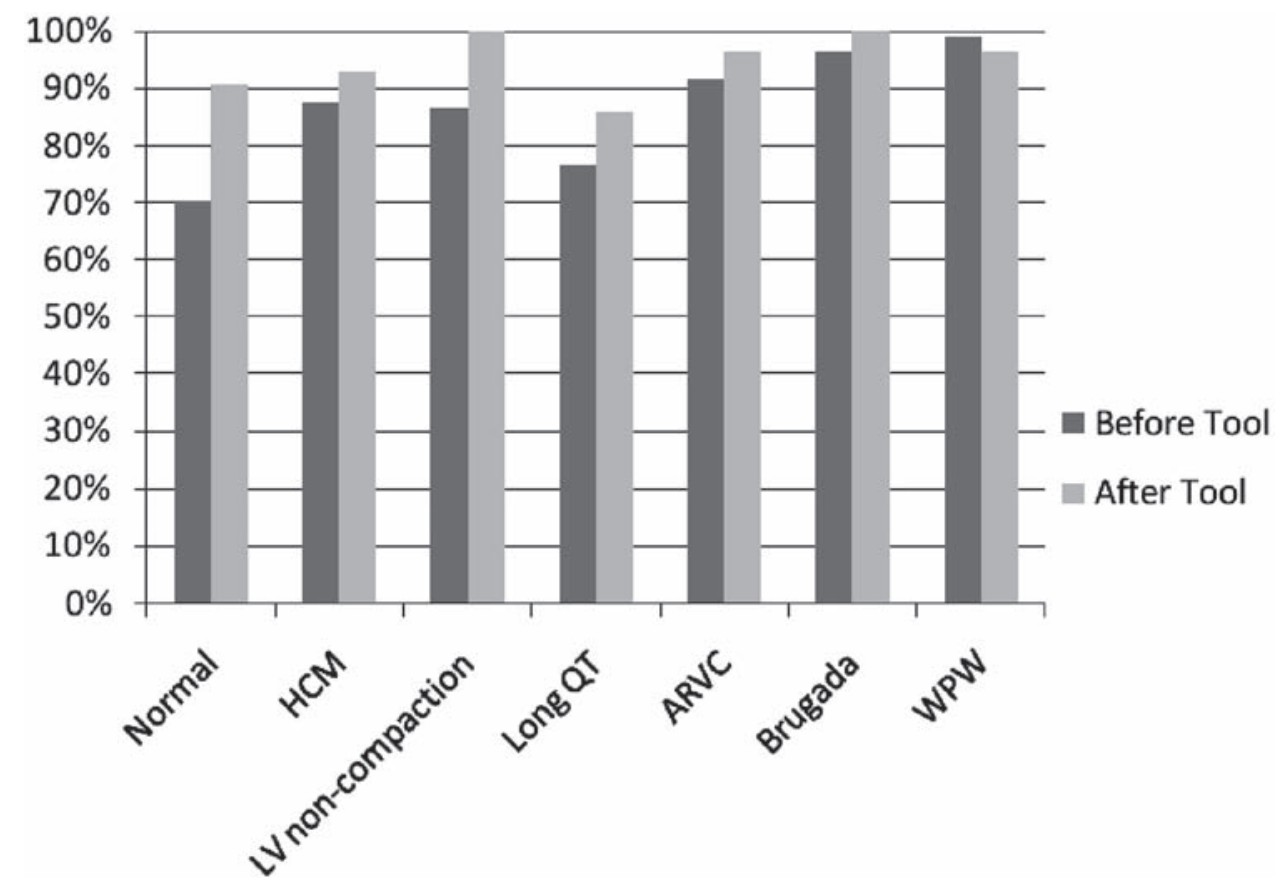

Figure 2 Accuracy of ECG interpretation by diagnosis. ARVC, arrhythmogenic right ventricular cardiomyopathy; HCM, hypertrophic cardiomyopathy; LV, left ventricular; WPW, Wolff-Parkinson-White.

abnormalities from physiological ECG alterations in athletes. ${ }^{4}$ In 2011, a US led statement on ECG interpretation in athletes provides additional detail and a description of contemporary ECG criteria and recommendations for secondary evaluations of abnormal findings. ${ }^{8}$

The most significant change from past ECG guidelines is the elimination of isolated ORS voltage criteria for left ventricular hypertrophy (LVH) as a cause for further evaluation. Isolated voltage criterion for $\mathrm{LVH}$ is an insensitive marker for $\mathrm{LVH}$, found in up to $40 \%$ of highly trained athletes but in less than $2 \%$ of patients with HCM. ${ }^{22}$ Isolated increases in ORS amplitude are common in trained athletes but have not been found to be associated with a diagnosis of HCM in young adults undergoing preparticipation screening. ${ }^{23}$ In contrast, non-voltage criteria for LVH such as atrial enlargement, left axis deviation, a 'strain' pattern of repolarisation, ST-segment depression, $\mathrm{T}$-wave inversion or pathological $\mathrm{Q}$ waves are considered abnormal and require further evaluation. ${ }^{4}$

Despite the publication and promotion of consensus guidelines for ECG interpretation, the impact of providing standardised criteria during ECG interpretation has not been previously studied. ECG training is a core part of medical education; however, ECG training specific to athletes is rare, and standards to optimise training and the best way to achieve and maintain competency are not known and largely consensus-based. ${ }^{24} 25$

This study showed that simply providing physicians with a standardised criteria tool, with no other training or information, significantly improves the ability for physicians to accurately distinguish normal from abnormal ECG findings in competitive athletes. Physicians were asked to correctly classify an ECG as normal (requiring no further testing) or abnormal (requiring further testing). Improvements in ECG interpretation with use of a criteria tool were seen across physician specialties despite these physicians having little or no experience in ECG interpretation in athletes. Physicians were not asked to make a specific diagnosis using only the ECG, although in some cases of ion channel disorders or ventricular pre-excitation this may have been possible. In the setting of abnormal findings suggestive of cardiomyopathy, a definitive diagnosis is rarely made on the basis of ECG alone and usually requires additional investigations and collaborating history.

Hill et $a^{26}$ examined the accuracy of paediatric cardiologists in interpreting an athlete's ECGs. Fifty-three participants reviewed 18 ECGs, 8 from patients with normal hearts and 10 from patients with conditions known to cause SCD. Participants were asked to fill in the blank and provide the diagnosis as their primary measure of accuracy. Only 69\% of ECGs were given the correct diagnosis leading study authors to conclude that mistakes in ECG interpretation could lead to high rates of inappropriate diagnostic testing and sports restriction. ${ }^{26}$ However, certain diagnoses, such as HCM and myocarditis, cannot be made on ECG alone, and this may have biased the results towards a lower accuracy. In fact, in the case of ECGs representing HCM, $80 \%$ of the participants would have restricted these players from play and $85 \%$ would have ordered an echocardiogram based on the ECG, even though only $59 \%$ could provide the correct diagnosis based on ECG alone. ${ }^{26}$ The correct follow-up testing recommended by participants in the Hill study is similar to the baseline accuracy of cardiologists (85\%) in our study before use of the criteria tool. Thus, the 'accuracy rate' referred to in the study by Hill et al may be an inaccurate representation of a physician's ability to interpret an athlete's ECG. A physician may not be able to correctly identify a specific diagnosis on ECG but may still realise that additional testing or consultation is warranted.

This study and the study by Hill et al demonstrated the need for improvement in ECG interpretation even among experts and the importance of using standardised criteria to guide the distinction between physiological changes and findings suggestive of pathology. It is not surprising that without a reference range or framework to judge an athlete's ECG that correct interpretation is challenging, similar to the difficulty in interpreting an unfamiliar laboratory value without having 
the reference range of normal values. ECG interpretation in athletes is complicated by physiological ECG changes that overlap with abnormal findings found in some cardiovascular disorders. In this study, the most common ECGs that were incorrectly classified were normal or normal variant ECGs from competitive athletes. Thirty per cent of normal ECGs were incorrectly classified as abnormal without use of the criteria tool. After provision of standardised criteria and with no additional teaching, normal ECGs that were incorrectly interpreted decreased to $9 \%$.

The debate regarding ECG screening in young athletes remains quite polarised. Major criticisms against ECG screening have been that testing cannot be done in large scale and that the false-positive rate will be too high (in the range of 10-40\%) leading to unnecessary diagnostic testing and restriction from athletic participation. An initial screening study performed in the USA over two decades ago reported a false-positive rate of $15 \% .{ }^{9}$ However, more recent studies applying modern ECG criteria to screen athletes have resulted in substantially lower false-positive rates ranging as low as $2-5 \% .^{7} 1011$

The cost-effectiveness of ECG screening is also affected by the use of modern ECG criteria to guide interpretation. In a study of cardiovascular screening in college athletes, the overall cost per diagnosis including all secondary evaluations was similar between history and physical compared with ECG. ${ }^{27}$ However, ECG interpretation was based on 2005 guidelines. ${ }^{3}$ If the study applied modern ECG criteria to assist in distinguishing physiological adaptations of an athlete's heart from findings suggestive of a pathological cardiac condition, ${ }^{4}$ it is possible the ECG false-positive rate would be reduced by $85 \%$ (from 19\% to $<3 \%$ ) with a correlating cost reduction simply by excluding voltage only and incomplete right bundle branch block findings as indications for additional investigation.

Similarly, in a study by Baggish et al, 510 college athletes were screened with history, physical examination, ECG and echocardiography. ${ }^{17}$ ECG improved the sensitivity for detecting important cardiac abnormalities from $45 \%$ to $91 \%$, but the study also reported a high false-positive ECG rate of $16 \% .{ }^{17}$ However, application of the 2010 ESC criteria reduced the number of abnormal ECG findings from $16 \%$ to $9 \% .{ }^{28}$ The improved specificity (reduction in false-positive rate) was largely driven by the reclassification of isolated ORS voltage criteria for LVH from abnormal to normal.

This study is limited by the small physician sample that interpreted the ECGs and that there was no method to ensure that the criteria tool was being used appropriately or referred to when needed. This study was purposefully conducted in physicians with little or no experience in ECG interpretation in athletes, and it is possible that a similar exercise in experienced physicians would yield greater accuracy. The study also does not account for testing behaviour and the possibility that physicians may interpret ECGs differently in a clinical setting where consequences exist if a clinically relevant finding is missed. Although physicians reviewed the same set of ECGs twice, there is little reason to believe that improvements in accuracy were related to a testing effect when the only change was the availability of a standardised ECG criteria tool. In addition, this study had a very high proportion of abnormal ECGs $(12 / 40)$, and the sensitivity and specificity may be different in a clinical setting with a lower frequency of true disease.

This study demonstrates that without further education, the baseline ability of physicians with little or no experience in interpreting an athlete's ECG is relatively poor and would lead to an unacceptable amount of secondary evaluations at a

\section{What is already known on this topic}

- ECG interpretation in athletes is complicated by ECG changes from physiological cardiac adaptations that overlap with abnormal ECG findings found in some cardiovascular disorders.

- Modern standards for ECG interpretation exist to help distinguish normal ECG changes in athletes from abnormalities suggestive of underlying pathology.

\section{What this study adds}

- In physicians with little experience in ECG interpretation in athletes, a large proportion of normal ECGs in athletes are incorrectly classified as abnormal.

- Use of standardised criteria to assist ECG interpretation significantly improves physician accuracy to distinguish normal from abnormal findings across physician specialties.

- Physician education in modern standards for ECG interpretation in athletes may improve the cardiovascular care of athletes by limiting false-positive results and enhancing disease detection.

significant cost. However, simply providing physicians with standardised criteria set with which to evaluate an ECG significantly improves accuracy. While this is only the first step in understanding the needs and potential for larger infrastructure development for the interpretation of ECGs in athletes, this study establishes that physician education in ECG interpretation is feasible and accompanied by significant improvements in all statistical measures of performance when a reference standard is used to guide interpretation.

\section{CONCLUSIONS}

Prevention of SCD in athletes remains a highly visible topic in cardiology and undoubtedly more research on cardiovascular screening and ECG interpretation in young athletes is needed. It is critical that physicians of any specialty be guided by standards that improve disease detection and limit false-positive results. A simple two-page criteria tool using modern standards for ECG interpretation significantly improves accuracy, even in physicians with little or no experience. Understanding the value of using a reference standard is only the first step towards improved ECG interpretation. Greater efforts towards physician education and formal training are needed to produce even better results leading to improved care of athletes.

Contributors All authors contributed to the study design, data analysis, writing and/or review of this manuscript.

Competing interests None.

Ethics approval University of Washington Division of Human Subjects.

Provenance and peer review Not commissioned; externally peer reviewed.

\section{REFERENCES}

1. Corrado D, Basso C, Pavei A, et al. Trends in sudden cardiovascular death in young competitive athletes after implementation of a preparticipation screening program. JAMA 2006;296:1593-601.

2. Corrado D, Biffi A, Basso C, et al. 12-lead ECG in the athlete: physiological versus pathological abnormalities. Br J Sports Med 2009;43:669-76. 
3. Corrado D, Pelliccia A, Bjørnstad HH, et al. Cardiovascular pre-participation screening of young competitive athletes for prevention of sudden death: proposal for a common European protocol. Consensus Statement of the Study Group of Sport Cardiology of the Working Group of Cardiac Rehabilitation and Exercise Physiology and the Working Group of Myocardial and Pericardial Diseases of the European Society of Cardiology. Eur Heart J 2005;26:516-24.

4. Corrado D, Pelliccia A, Heidbuchel $\mathrm{H}$, et al. Recommendations for interpretation of 12-lead electrocardiogram in the athlete. Eur Heart J 2010;31:243-59.

5. Drezner JA. Contemporary approaches to the identification of athletes at risk for sudden cardiac death. Curr Opin Cardiol 2008;23:494-501.

6. Drezner J, Berger S, Campbell R. Current controversies in the cardiovascular screening of athletes. Curr Sports Med Rep 2010;9:86-92.

7. Pelliccia A, Culasso F, Di Paolo FM, et al. Prevalence of abnormal electrocardiograms in a large, unselected population undergoing pre-participation cardiovascular screening. Eur Heart J 2007;28:2006-10.

8. Uberoi A, Stein R, Perez MV, et al. Detailed criteria for the interpretation of the electrocardiogram in young athletes. Circulation 2011;124:746-57.

9. Maron BJ, Bodison SA, Wesley YE, et al. Results of screening a large group of intercollegiate competitive athletes for cardiovascular disease. J Am Coll Cardiol 1987; 10:1214-21.

10. Wilson MG, Basavarajaiah S, Whyte GP, et al. Efficacy of personal symptom and family history questionnaires when screening for inherited cardiac pathologies: the role of electrocardiography. Br J Sports Med 2008;42:207-11.

11. Marek J, Bufalino V, Davis J, et al. Feasibility and findings of large-scale electrocardiographic screening in young adults: data from 32,561 subjects. Heart Rhythm 2011;8:1555-9

12. Ljungqvist $\mathbf{A}$, Jenoure $P$, Engebretsen $L$, et al. The International Olympic Committee (IOC) consensus statement on periodic health evaluation of elite athletes March 2009. Br J Sports Med 2009;43:631-43.

13. Maron BJ, Thompson PD, Ackerman MJ, et al. Recommendations and considerations related to preparticipation screening for cardiovascular abnormalities in competitive athletes: 2007 update: a scientific statement from the American Heart Association Council on Nutrition, Physical Activity, and Metabolism: endorsed by the American College of Cardiology Foundation. Circulation 2007;115:1643-455

14. Harmon KG, Asif IM, Klossner D, et al. Incidence of sudden cardiac death in national collegiate athletic association athletes. Circulation 2011;123:1594-600

15. Maron BJ. Sudden death in young athletes. N Engl J Med 2003;349:1064-75
16. Maron BJ, Doerer JJ, Haas TS, et al. Sudden deaths in young competitive athletes: analysis of 1866 deaths in the United States, 1980-2006. Circulation 2009;119:1085-92.

17. Baggish AL, Hutter AM Jr, Wang F, et al. Cardiovascular screening in college athletes with and without electrocardiography: A cross-sectional study. Ann Intern Med 2010;152:269-75.

18. Hevia AC, Fernández MM, Palacio JM, et al. ECG as a part of the preparticipation screening programme: an old and still present international dilemma. Br J Sports Med 2011;45:776-9.

19. Bessem B, Groot FP, Nieuwland W. The Lausanne recommendations: a Dutch experience. Br J Sports Med 2009;43:708-15

20. Drezner J, Corrado D. Is there evidence for recommending electrocardiogram as part of the pre-participation examination? Clin J Sport Med 2011;21:18-24.

21. Thompson PD, Levine BD. Protecting athletes from sudden cardiac death. JAMA 2006;296:1648-50.

22. Melacini P, Cianfrocca C, Calore C, et al. Abstract 3390: Marginal overlap between electrocardiographic abnormalities in patients with hypertrophic cardiomyopathy and trained athletes: implications for preparticipation screening. Circulation 2007;116:765

23. Sathanandam S, Zimmerman F, Davis J, et al. Abstract 2484: ECG screening criteria for LVH does not correlate with diagnosis of hypertrophic cardiomyopathy. Circulation 2009:S647.

24. Salerno SM, Alguire PC, Waxman HS. Training and competency evaluation for interpretation of 12-lead electrocardiograms: recommendations from the American College of Physicians. Ann Intern Med 2003;138:747-50.

25. Kadish AH, Buxton AE, Kennedy HL, et al. ACC/AHA clinical competence statement on electrocardiography and ambulatory electrocardiography: A report of the ACC/AHA/ACP-ASIM task force on clinical competence (ACC/AHA Committee to develop a clinical competence statement on electrocardiography and ambulatory electrocardiography) endorsed by the International Society for Holter and noninvasive electrocardiology. Circulation 2001;104:3169-78.

26. Hill AC, Miyake CY, Grady S, et al. Accuracy of interpretation of preparticipation screening electrocardiograms. J Pediatr 2011;159:783-8.

27. Malhotra R, West JJ, Dent J, et al. Cost and yield of adding electrocardiography to history and physical in screening Division I intercollegiate athletes: a 5-year experience. Heart Rhythm 2011;8:721-7.

28. Weiner RB, Hutter AM, Wang F, et al. Performance of the 2010 European Society of Cardiology criteria for ECG interpretation in athletes. Heart 2011;97:1573-7. 